\title{
Оценка величины модуля вектора магнитного поля из $I$ и $V$ компонент вектора Стокса
}

\author{
А.А. Плотников, А.С. Куценко \\ ФГБУН “Крымская астрофизическая обсерватория РАН”, Научный, Крым, 298409 \\ plotnikov.andrey.alex@yandex.ru
}

Поступила в редакцию 27 июня 2018 г.

\begin{abstract}
Аннотация. В данной работе мы описываем простой метод оценки абсолютной величины магнитного поля только из $I$ и $V$ компонент вектора Стокса. Модуль вектора магнитного поля может быть определен из положений экстремумов профиля $V$ компоненты вектора Стокса. Также, оценив продольное магнитное поле в приближении слабого поля, можно легко определить модуль поперечного магнитного поля. Предлагаемый метод протестирован на спектрополяриметрических наблюдениях космической обсерватории Hinode и показал хорошее согласие между нашими оценками полей и результатами измерения магнитных полей более сложными методами, основанными на решении уравнения переноса излучения в присутствии магнитного поля.

DERIVING ABSOLUTE VALUE OF THE MAGNETIC FIELD STRENGTH FROM $I$ AND $V$ STOKES PARAMETERS, by A.A. Plotnikov, A.S. Kutsenko. In this work, we describe a simple technique to evaluate the absolute value of the magnetic field strength from $I$ and $V$ Stokes parameters. The absolute value of the vector magnetic field can be derived from the extrema positions of Stokes $V$ profile. One can simply evaluate the modulus of the transversal magnetic field from these two quantities. The spectropolarimetric data acquired by Hinode were used to test the technique proposed. Our results are in a good agreement with the magnetic field measurements provided by the Hinode team.
\end{abstract}

Ключевые слова: магнитное поле, Солнце, активная область

\section{1 Введение}

Магнетизм играет определяющую роль в процессах, генерирующих явления солнечной активности. Наблюдаемые проявления солнечной активности - пятна, корональные дыры, вспышки, протуберанцы, корональные выбросы массы - связаны с выходом магнитного потока из глубины конвективной зоны на поверхность Солнца (Паркер, 1955). Установлено, что циклы магнитной активности коррелируют с изменением полного солнечного излучения (Йео и др., 2017), поэтому длительные перерывы в магнитной активности Солнца могут приводить к существенным изменениям климата на Земле, как, например, во время знаменитого минимума Маундера. С другой стороны, проявления большой солнечной активности, такие как мощные вспышки или корональные выбросы массы, могут негативно влиять на магнитосферу Земли, приводя к перебоям в работе связи, выходу из строя техники, 
электрогенерирующих мощностей и др. Для минимизации негативных последствий необходимо заблаговременно предсказывать такие события как на длительных интервалах времени (глобальные минимумы), так и на малых (вспышки, солнечный ветер и корональные выбросы массы). Прогноз солнечной активности, основанный на физических законах, подразумевает создание модели солнечного магнетизма.

После открытия Хейлом сильных магнитных полей в солнечных пятнах в начале прошлого века (Хейл, 1908) и появления первых феноменологических моделей солнечного динамо, например, модели Бэбкока-Лейтона (Бэбкок, 1961; Лейтон, 1969), с развитием вычислительной техники наблюдается существенный прогресс в моделировании солнечного и звездного динамо (Нельсон и др., 2013; Фан, Фань, 2014). Естественно, правомочность любой модели определяется ее способностью описывать реально наблюдаемые явления, поэтому модели солнечного динамо должны верифицироваться сравнением результатов моделирования с реально наблюдаемыми на поверхности Солнца магнитными полями. Это, конечно, требует усовершенствования инструментов и методов для измерения магнитных полей в атмосфере Солнца.

Основным методом измерения магнитных полей на Солнце является спектрополяриметрический метод, основанный на эффекте Зеемана. Эффект Зеемана заключается в расщеплении спектральных уровней излучающих или поглощающих атомов на несколько подуровней при помещении атомов во внешнее магнитное поле. Вследствие расщепления энергетических уровней, в спектре наблюдается расщепление спектральной линии на несколько компонент. Следует особо отметить, что величина расщепления энергетических уровней для конкретного перехода (мы не рассматриваем зависимость расщепления от наборов квантовых чисел верхнего и нижнего уровней) зависит только от абсолютной величины (модуля вектора) магнитного поля (см., например, Дегл'Инносенти и Ландолфи, 2004 и ссылки в ней). При этом поляризация излучения определяется направлением магнитного поля по отношению к лучу зрения. В простейшем случае расщепления синглетной спектральной линии на триплет, при строго продольном магнитном поле в спектре будут наблюдаться две $\sigma$-компоненты с круговой поляризацией, их смещения относительно центра нерасщепленной линии определяются абсолютной величиной магнитного поля. При строго поперечном поле положение в спектре $\sigma$-компонент с линейной поляризацией будет таким же, как и в предыдущем случае, при этом уменьшится их амплитуда, поскольку часть энергии перейдет в $\pi$-компоненту. Таким образом, взаимная ориентация направления на наблюдателя и вектора магнитного поля будет определять только перераспределение энергии между излучением с различными поляризациями, т. е. соотношение амплитуд $\pi$ - и $\sigma$ компонент зеемановского мультиплета, в то время как само расщепление (разность между длиной волны нерасщепленной компоненты и длинами волн $\pi$ - и $\sigma$-компонент) будет оставаться постоянным при неизменной величине магнитного поля. Из всего вышесказанного следует, что, если нам удастся каким-либо образом определить величину расщепления компонент из наблюдаемого спектра, мы сможем измерить абсолютную величину магнитного поля. Последнее может быть актуально в связи с тем, что прецизионные измерения линейно-поляризованной компоненты в электромагнитном излучении связаны с существенными инструментальными трудностями по сравнению с измерением поляризованной по кругу компоненты. Поэтому первые магнитографы проводили измерения только общей интенсивности излучения и поляризованного по кругу излучения, т. е. $I$ и $V$ параметров Стокса. Вследствие этого, до появления наблюдений современных спектрополяриметров, основной измеряемой на Солнце магнитографической величиной была продольная компонента магнитного поля.

Цель данной работы - показать, что оценка величины модуля вектора магнитного поля может быть дана только на основании наблюдаемых $I$ и $V$ параметров Стокса с помощью простого метода. Подобные исследования выполнялись раньше, см., например, работы Рэйроля (1967) или Брантса (1985). Метод может применяться в случае, если для анализа доступны только наблюдения поляризованного по кругу излучения, что до сих пор актуально для некоторых инструментов, например, для магнитографа обсерватории Маунт-Вилсон (Ульрих и др., 2002). Также данный метод может быть применен к первым наблюдениям нового солнечного магнитографа KрAО, который в настоящее время позволяет проводить измерения солнечного спектра в поляризованном по кругу излучении. 


\section{2 Данные и методы}

\section{1 Данные}

В данной работе мы использовали наблюдения инструмента Spectropolarimeter - Solar Optical Telescope (SOT-SP) внеатмосферной обсерватории Hinode. Спектрополяриметр Hinode/SOT-SP представляет собой щелевой спектрограф с высотой щели 150". Для измерений магнитных полей используются линии Fe I $6301.5 \AA$ и $6302.5 \AA$. Максимальное поле зрения инструмента составляет $150^{\prime \prime} \times 300^{\prime \prime}$ с пространственным разрешением $0.16^{\prime \prime}$ и с разрешением по длине волны $21.5 \mathrm{~m} \AA$. Поляризационный шум составляет $\sim 10^{-3}$ от интенсивности континуума (Лайтс и др., 2013).

Готовые для научного анализа спектрополяриметрические наблюдения Hinode находятся в свободном доступе на сайте обсерватории High Altitude Observatory (HAO) ${ }^{1}$. Данные представляют собой измеренные профили параметров Стокса для каждого положения щели спектрографа. Для определения продольного поля и модуля вектора магнитного поля мы использовали измерения только $I$ и $V$ параметров Стокса для спектральной линии Fe I $6302.5 \AA$.

Для проверки правомочности наших методов мы провели сравнение наших результатов с результатами измерения полного вектора магнитного поля, полученными в НАО. Карты вектора магнитного поля, а также ряда других величин, таких как доплеровские скорости, интенсивность в континууме и др., определяются командой НАО из наблюдаемых параметров Стокса с помощью алгоритма MERLIN (Лайтс и др., 2007). Алгоритм проводит аппроксимацию наблюдаемых профилей параметров Стокса модельными профилями в приближении атмосферы Милна - Эддингтона, подбирая значения модельных параметров (магнитное поле, доплеровская скорость, функция источника, доплеровское уширение и др.). Вектор магнитного поля представлен в виде карт абсолютной величины $|\mathbf{B}|$, наклона к лучу зрения $\theta$ и азимута вектора магнитного поля. Модуль вектора магнитного поля $|\mathbf{B}|$, продольное магнитное поле $B_{z}=|\mathbf{B}| \cos \theta$ и модуль поперечного магнитного поля $\left|\mathbf{B}_{t}\right|=|\mathbf{B}| \sin \theta$, полученные группой обсерватории $\mathrm{HAO}$ и используемые в данной работе для сравнения с нашими расчетами, мы будем далее обозначать как $\left|\mathbf{B}^{H i n}\right|, B_{z}^{H i n}$ и $\left|\mathbf{B}_{t}^{H i n}\right|$ соответственно.
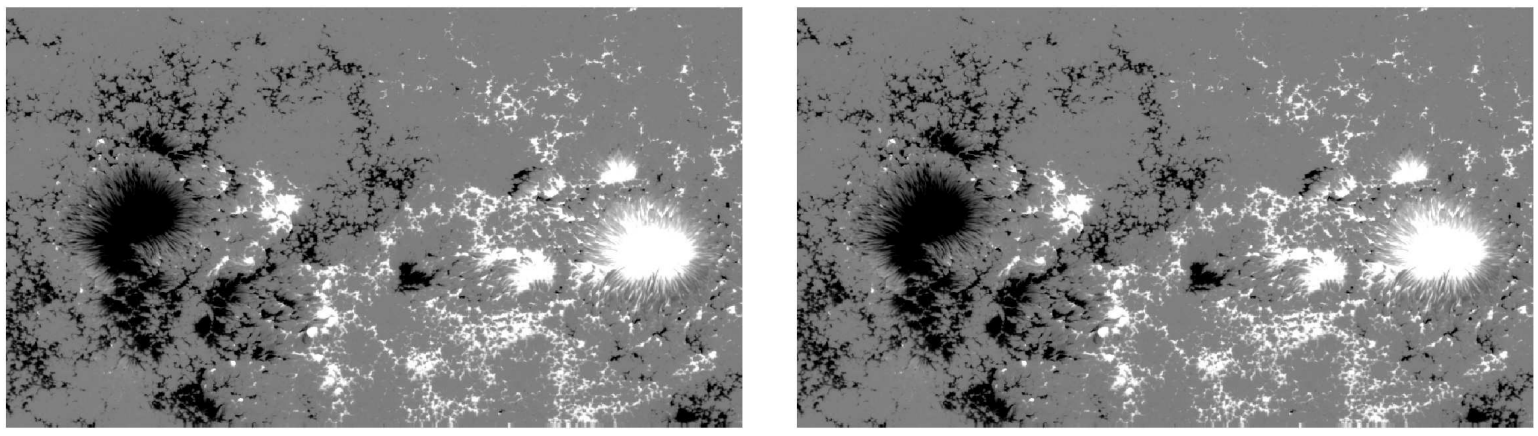

Рис. 1. Магнитограммы продольного магнитного магнитного поля AO NOAA 12172 (время наблюдений 26 сентября 2014 года в 17:00 UT). Слева - продольное магнитное поле, полученное группой обсерватории НАО, справа - продольное магнитное поле, рассчитанное в данной работе в приближении слабого поля (см. текст). Поле зрения магнитограмм $250^{\prime \prime} \times 150^{\prime \prime}$. Черный цвет соответствует величине поля -1000 Гс, белый цвет - 1000 Гс

В данной работе мы использовали результаты наблюдений активных областей (AO) NOAA 12172 и 12645, полученные 26 сентября 2014 года в 17:00 UT и 1 апреля 2017 года в 11:00 UT соответственно. Обе АО в выбранные моменты времени находились в центре видимого диска, поэтому

${ }^{1}$ https://www2 hao.ucar.edu/csac/csac-data 
мы полагаем, что луч зрения совпадает с $z$-направлением в локальной системе координат. АО представляли собой магнитные диполи с несколькими хорошо сформированными пятнами в ведущей и хвостовой полярностях. Магнитограммы продольного поля АO NOAA 12172 показаны на рис. 1.

\section{2 Определение продольной компоненты магнитного поля}

Определение магнитного поля из наблюдаемого зеемановского мультиплета в общем случае требует решения уравнения переноса излучения в солнечной атмосфере при наличии магнитного поля (например, Дегл'Инносенти и Ландолфи, 2004). В то же время в небольших по величине магнитных полях, когда зеемановское расщепление существенно меньше доплеровского уширения линии, для оценки магнитного поля возможно использовать приближение слабого поля (Дегл'Инносенти и Ландолфи, 2004). В рамках приближения слабого поля величина $V$ параметра Стокса пропорциональна продольной составляющей $B_{z}=|\mathbf{B}| \cos \theta$ магнитного поля:

$$
V(\lambda)=-\frac{d I}{d \lambda} g \Delta \lambda \cos \theta=-\frac{d I}{d \lambda} g \frac{\lambda^{2} e}{4 \pi m_{e} c^{2}} B_{z}
$$

где $\lambda$ - длина волны несмещенной линии, $\Delta \lambda$ - величина расщепления спектральной линии вследствие эффекта Зеемана, $g$ - фактор Ланде, e и $m_{e}-$ заряд и масса электрона, $c$ - скорость света, $\cos \theta$ - угол между вектором магнитного поля и направлением к наблюдателю. Величина поля, до которой сохраняется линейная зависимость, определяется параметрами спектральной линии. Так, для спектральной линии, используемой в этой работе, она составляет около 2500 Гс. Посредством интегрирования выражение (1) можно привести к более удобному виду:

$$
\int_{a}^{b} V(\lambda) d \lambda=-(I(b)-I(a)) g \frac{\lambda^{2} e}{4 \pi m_{e} c^{2}} B_{z} .
$$

Фактор Ланде $g$, входящий в выражения 1-2, следует определять экспериментальным путем, поскольку он может сильно отличаться от теоретического при нарушении $L S$-связи в атоме (см., например, Дегл'Инносенти, 1982; Соланки и Стэнфло, 1985). По этой причине мы вводим калибровочную константу для продольного поля $C_{z}=g \frac{\lambda^{2} e}{4 \pi m_{e} c^{2}}$, которую можно подобрать, например, путем кросскалибровки двух инструментов. Этот прием широко используется при реальных измерениях магнитных полей. В данной работе мы подбираем $C_{z}$ таким образом, чтобы рассчитанное нами продольное магнитное поле

$$
B_{z}=C_{z} \frac{\int_{a}^{b} V(\lambda) d \lambda}{(I(a)-I(b))}
$$

наилучшим образом соответствовало $B_{z}^{\text {Hin }}$.

Численно величина продольного магнитного поля $B_{z}$ определялась из $(3)$ как площадь под кривой параметра $V$, нормированная на максимальную амплитуду параметра $I$ Стокса, $I(b)-I(a)$, что схематически показано на рис. 2. Для определения площади под кривой параметра $V$ (выделена серым и красным цветом на рис. 2), мы вычисляли интеграл $\int_{a}^{b} V(\lambda) d \lambda-\int_{b}^{c} V(\lambda) d \lambda$, где пределы интегрирования $a$ и $c$ выбирались на границах спектрограммы, а точка $b$ соответствовала минимуму параметра Стокса $I$. 


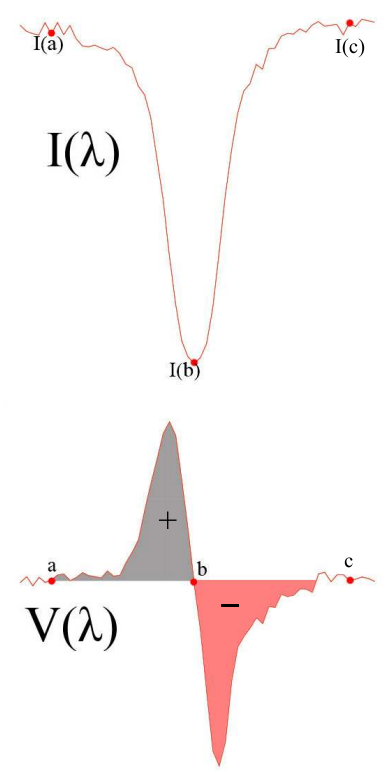

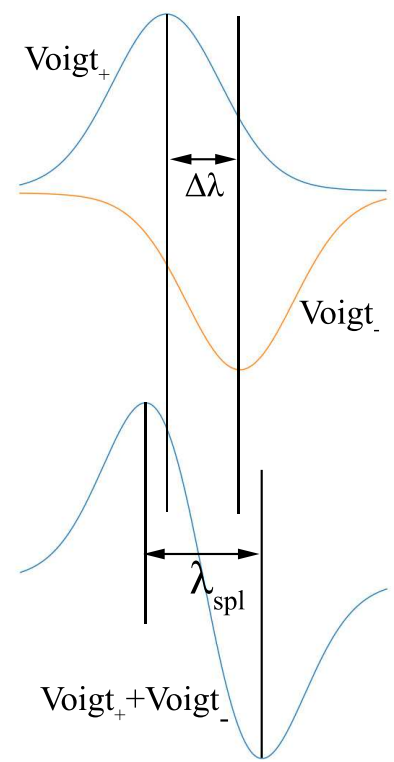

б

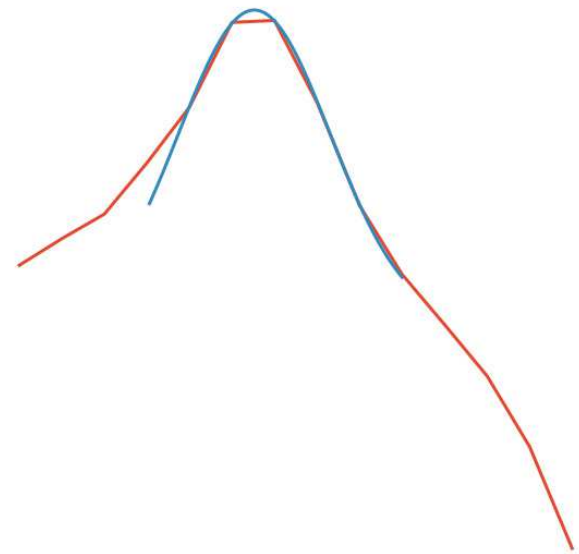

B

Рис. 2. а - Объяснение к формуле (3) для определения продольного магнитного поля. Площадь заштрихованных областей под профилем $V$ параметра Стокса пропорциональна продольному магнитному полю. Пределы интегрирования $a$ и $c$ выбираются на краях спектрограммы, а точка $b$ - в минимуме $I$ параметра Стокса. б Схематическое объяснение смещений положений экстремумов $V$ параметра Стокса вследствие блендирования спектральных линий. На рисунке показано фактическое расщепление спектральных линий $\Delta \lambda$ и его проксивеличина (величина, похожая по характеру на искомую величину, но более простая в нахождении) $\lambda_{s p l}$, определяемая в данной работе. в - Пример аппроксимации одного из экстремумов $V$ параметра Стокса (красная кривая) гауссианой (синяя кривая) для определения положения экстремума с субпиксельной точностью

\section{3 Определение модуля вектора магнитного поля}

Расщепление между спектральными компонентами в зеемановском мультиплете пропорционально модулю вектора магнитного поля (например, Дегл’Инносенти и Ландолфи, 2004):

$$
\Delta \lambda=g \frac{\lambda^{2} e}{4 \pi m_{e} c^{2}}|\mathbf{B}| .
$$

Как упоминалось выше, расщепление спектральных линий связано с расщеплением энергетических уровней атома, и, следовательно, определяется только свойствами атома и абсолютной величиной поля. Изменение направления вектора магнитного поля относительно луча зрения не оказывает влияния на сдвиг энергетических уровней атома. Следует заметить, что ориентация вектора магнитного поля относительно направления к наблюдателю определяет только поляризацию и амплитуду спектральных компонент, а не положение их центров на оси длин волн.

Величину расщепления спектральных линий можно грубо определить путем измерения разности длин волн между максимумом и минимумом параметра $V$ (рис. 2б). Данный способ не совсем точен, т. к. положение экстремумов несколько сдвигается из-за блендирования линий и чрезмерно завышает оценку величины поля при $|\mathbf{B}|<1000$ Гс (Брантс, 1985). Пример такого сдвига для модельных профилей Фойгта показан на рис. 2б. Также в слабых полях сказывается малая интенсивность параметра $V$ на фоне шумов. Это приводит к проблеме поиска истинных пиков спектра на зашумленном фоне. Некоторый вклад в смещение пиков вносит и наклон магнитного поля к лучу зрения (Бекерс, 1969). Для полноценного определения расщепления линий (а вместе с ним - и магнитного поля) требуется 
аппроксимировать $V$ параметр Стокса разностью двух спектральных профилей. Однако и предлагаемый здесь упрощенный способ позволяет получить адекватную оценку величины магнитного поля, что будет показано ниже.

Для определения положений экстремумов $V$ параметра Стокса мы выполняли следующую процедуру. Алгоритм выделял все точки, значение функции $V$ в которых было больше (для минимумов - меньше) значений в четырех соседних точках. Поскольку, в первом приближении, расщепление должно быть симметрично относительно длины волны центра несмещенной линии, из полученного множества точек выбиралась такая пара “минимум-максимум”, которая была расположена наиболее симметрично относительно несмещенной линии. Дискретность измерения длины волны приводит к тому, что положение найденных таким образом экстремумов будет также меняться дискретно - на всю ширину линии Fe I $6302.5 \AA$ в измерениях Hinode/SOT-SP приходится приблизительно 30 точек. Поэтому, для определения положений экстремумов с субпиксельной точностью, экстремум и соседние 10 точек аппроксимировались гауссианой, вершина которой принималась за фактическое положение экстремума (см. рис. 2в). Разность длин волн экстремумов профиля $V$ параметра Стокса мы принимаем за некую прокси-величину модуля вектора магнитного поля и будем обозначать ее $\lambda_{s p l}$ (рис. 2б).

Алгоритм определения положения экстремумов приводит к некорректным результатам, если профиль $V$ параметра Стокса слабо выделяется на фоне шумов, что наблюдается в слабых полях спокойного Солнца или в ядре сильных пятен, где недостаток фотонов приводит к сильному падению уровня сигнала. Чтобы избежать сильного влияния шумов на результаты, мы определяли модуль вектора магнитного поля по описанной выше методике только в тех пикселях магнитограммы, в которых уровень круговой поляризации превышал 1 \%, т. е. в которых

$$
V^{2} / I^{2} \geq 0.01 \text {. }
$$

Мы полагали магнитное поле равным нулю в тех пикселях, где это условие не выполнялось.

Имея измерения продольной компоненты магнитного поля $B_{z}$ и модуля вектора магнитного поля $|\mathbf{B}|$, мы можем легко получить оценку модуля поперечного магнитного поля $\left|\mathbf{B}_{t}\right|$ из простых геометрических соображений:

$$
\left|\mathbf{B}_{t}\right|=\left(|\mathbf{B}|^{2}-B_{z}^{2}\right)^{0.5}
$$

\section{3 Результаты}

Магнитограмма продольного поля $B_{z}$ AO NOAA 12172 , вычисленного в приближении слабого поля по формуле (3), показана на правой панели рис. 1. Видно хорошее визуальное согласие с магнитограммой продольного поля $B_{z}^{H i n}$, полученной группой обсерватории НАО. Хорошее согласие подтверждается диаграммой рассеяния $B_{z}$ vs. $B_{z}^{H i n}$, показанной на левой панели рис. 3 , коэффициент корреляции между величинами равен 0.99. Калибровочная константа в (3) подбиралась таким образом, чтобы коэффициент линейной регрессии между $B_{z}^{H i n}$ и $B_{z}$ был равен единице.

На правой панели рис. 3 показана зависимость между величиной $\lambda_{s p l}$, рассчитанной в пикселях магнитограммы, для которых выполнялось условие (5), и модулем вектора магнитного поля $\left|\mathbf{B}^{\text {Hin }}\right|$ в соответствующих пикселях для АO NOAA 12172. Как и в предыдущем случае, наблюдается высокая корреляция между величинами, коэффициент корреляции Пирсона равен 0.85. Видно, что облако точек группируется вокруг прямой, уравнение которой показано на правой панели рис. 3. Это, а также линейная зависимость между величиной расщепления спектральной линии и модулем вектора магнитного поля в выражении (4) (напомним, что $\lambda_{s p l}$ - это не само расщепление, а его проксивеличина), позволяет нам представить зависимость между $\lambda_{s p l}$ и $|\mathbf{B}|$ в виде

$$
|\mathbf{B}|=C_{t}^{1}+C_{t}^{2} \lambda_{s p l}
$$

где $C_{t}^{1}$ и $C_{t}^{2}$ - калибровочные константы для модуля вектора магнитного поля. То, что линейная аппроксимация распределения $\lambda_{s p l}$ vs. $|\mathbf{B}|$ не пересекает начало координат на рис. 3 , может быть объяснено смещением положений экстремумов профиля параметра $V$ из-за блендирования реально 

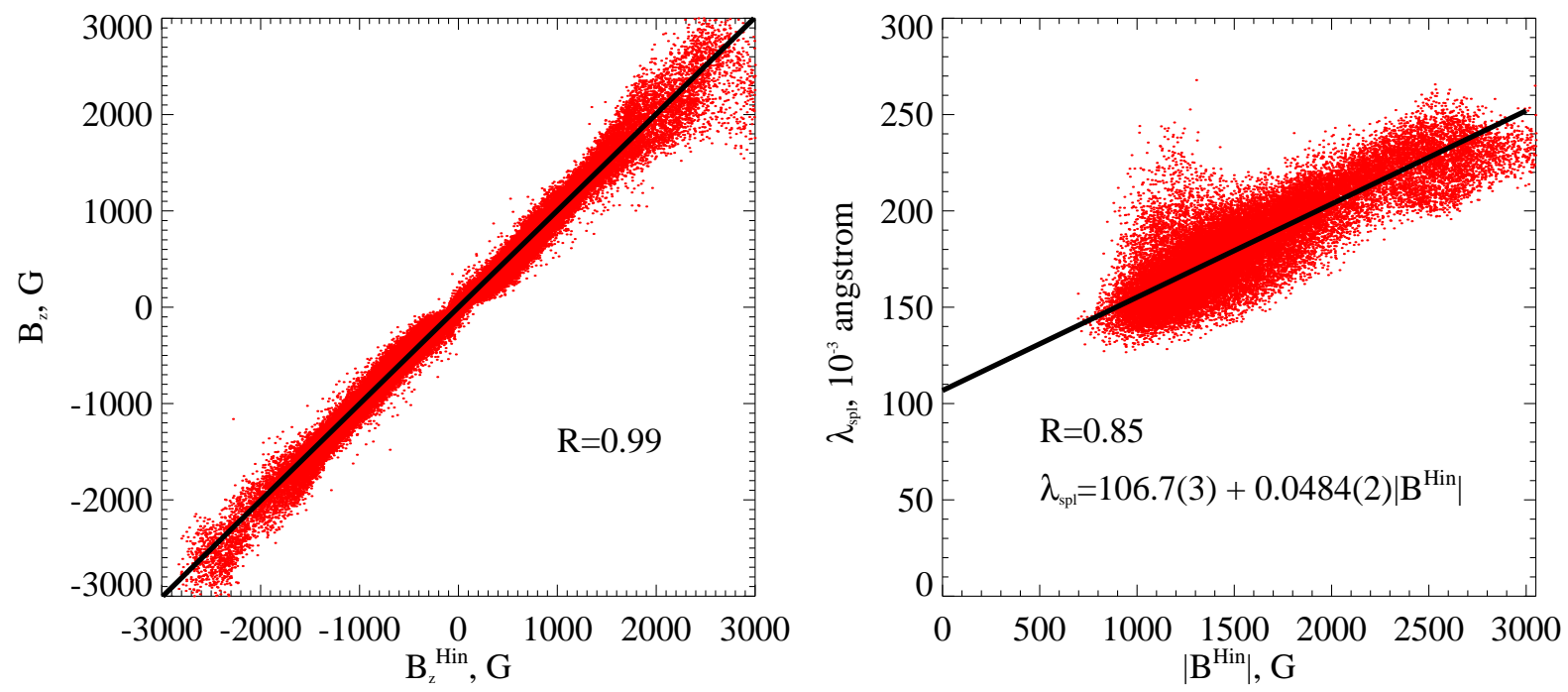

Рис. 3. Левая панель - диаграмма рассеяния продольного магнитного поля AO NOAA 12172, определенного группой обсерватории $\mathrm{HAO}\left(B_{z}^{H i n}\right)$ и в приближении слабого поля $\left(B_{z}\right)$. Черная сплошная прямая показывает наилучшую аппроксимацию распределения линейной функцией. Правая панель - зависимость величины $\lambda_{s p l}$ от абсолютной величины магнитного поля $\left|\mathbf{B}^{H i n}\right|$. Черная сплошная прямая показывает наилучшую аппроксимацию распределения линейной функцией. Уравнение прямой показано на рисунке

наблюдаемых при измерениях профилей $I+V$ и $I-V$ параметров Стокса, что ясно из рис. $2 б$. Действительно, величина $\lambda_{s p l}$ всегда будет больше реального расщепления $\Delta \lambda$.

Для проверки правомочности наших методов, используя калибровочные константы, определенные для AO NOAA 12172, нами проведены расчеты продольной компоненты и модуля вектора магнитного поля в AO NOAA 12645. Как и в предыдущем случае, мы сравниваем рассчитанные нами поля с результатами расчетов группы обсерватории $\mathrm{HAO}$, полагая, что калибровочные константы $C_{z}, C_{t}^{1}$ и $C_{t}^{2}$ зависят только от используемой для наблюдений спектральной линии и особенностей инструмента.

Зависимость рассчитанного нами продольного поля $B_{z}$ от $B_{z}^{\text {Hin }}$ по формуле (3) для тестовой $\mathrm{AO}$ NOAA 12645 показана на левой панели рис. 4. Видна очень хорошая корреляция между величинами, коэффициент корреляции Пирсона равен 0.99. Коэффициент линейной регрессии также близок к единице и равен 1.04. Магнитограммы продольного поля, показанные на рис. 5, также показывают хорошее визуальное согласие.

Распределение для модуля вектора магнитного поля для этой же АО показано на правой панели рис. 4. Для расчетов использовалось выражение (7) с константами $C_{t}^{1}$ и $C_{t}^{2}$, определенными для $\mathrm{AO}$ NOAA 12172. В этом случае также наблюдается хорошая корреляция (с коэффициентом корреляции 0.83), коэффициент линейной регрессии уже сильнее отличается от единицы и равен 0.79 .

Отсутствие точек с абсолютной величиной магнитного поля менее 700 Гс на правой панели рис. 4 объясняется критерием (5): слабые магнитные поля часто не показывают достаточного уровня сигнала с круговой поляризацией, чтобы наш алгоритм определения положений экстремумов $V$ параметра Стокса мог верно интерпретировать спектры.

На рис. 6 показано сравнение магнитограмм модуля вектора магнитного поля AO NOAA 12645. Левая панель показывает $\left|\mathbf{B}^{\text {Hin }}\right|$, правая - модуль вектора поля $|\mathbf{B}|$, рассчитанный в данной работе. Видно хорошее визуальное согласие обеих магнитограмм. Напомним, что пиксели магнитограммы, для которых не выполнялось условие (5), имеют нулевое значение $|\mathbf{B}|$. 

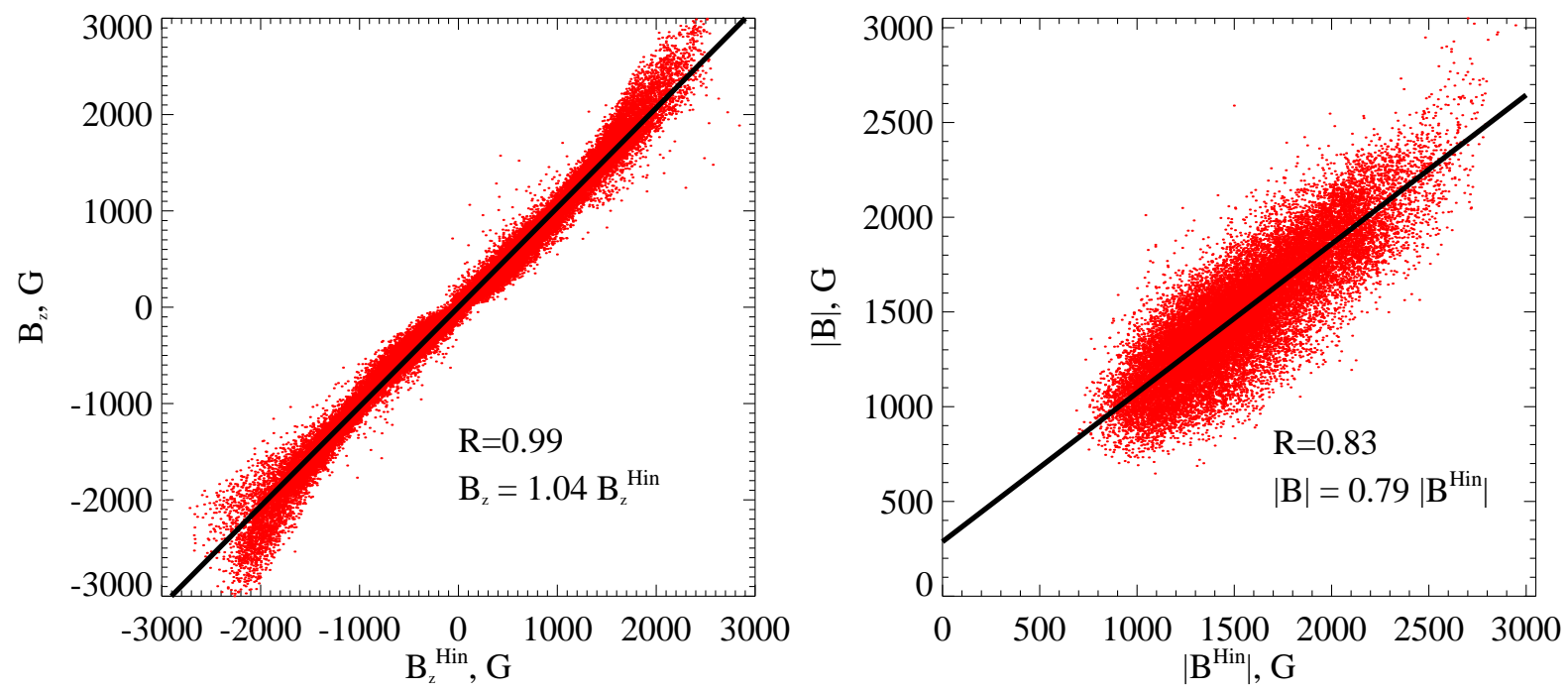

Рис. 4. Левая панель - диаграмма рассеяния продольного магнитного поля AO NOAA 12645, определенного группой обсерватории $\mathrm{HAO}\left(B_{z}^{H i n}\right)$ и в приближении слабого поля $\left(B_{z}\right)$. Черная сплошная прямая показывает наилучшую аппроксимацию распределения линейной функцией. Правая панель - диаграмма рассеяния модуля вектора магнитного поля AO NOAA 12645 , определенного группой обсерватории НАO $\left|\mathbf{B}^{\text {Hin }}\right|$ ) и в данной работе $(|\mathbf{B}|)$. Черная сплошная прямая показывает наилучшую аппроксимацию распределения линейной функцией
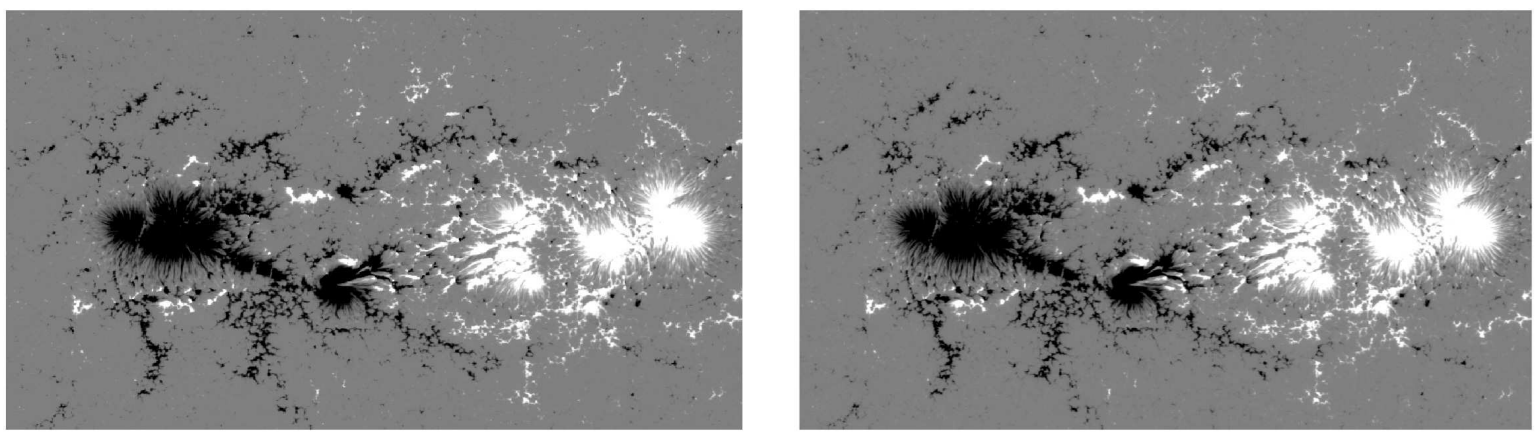

Рис. 5. Магнитограммы продольного магнитного поля тестовой АO NOAA 12645 (время наблюдений - 1 апреля 2017 года в 11:00 UT). Слева - продольное магнитное поле получено группой обсерватории НАО, справа продольное магнитное поле получено в данной работе в приближении слабого поля. Поле зрения магнитограмм $250^{\prime \prime} \times 150^{\prime \prime}$. Черный цвет соответствует величине поля -1000 Гс, белый цвет -1000 Гс

Наконец, на рис. 7 показаны магнитограммы модуля поперечного поля $\left|\mathbf{B}_{t}^{\text {Hin }}\right|$ (слева) и $\left|\mathbf{B}_{t}\right|$ (справа). Величина $\left|\mathbf{B}_{t}\right|$ рассчитывалась с помощью выражения (6). Из сравнения изображений следует, что также наблюдается хорошее визуальное согласие между величинами, повторяется пространственная структура поперечного поля. Примечательно, что на обеих магнитограммах различимы области относительно больших величин модуля поперечного магнитного поля в полутени пятен (к сожалению, из-за качества отображения магнитограмм, эти области слабо выделяются, на них указывают белые стрелки на рис. 7). 

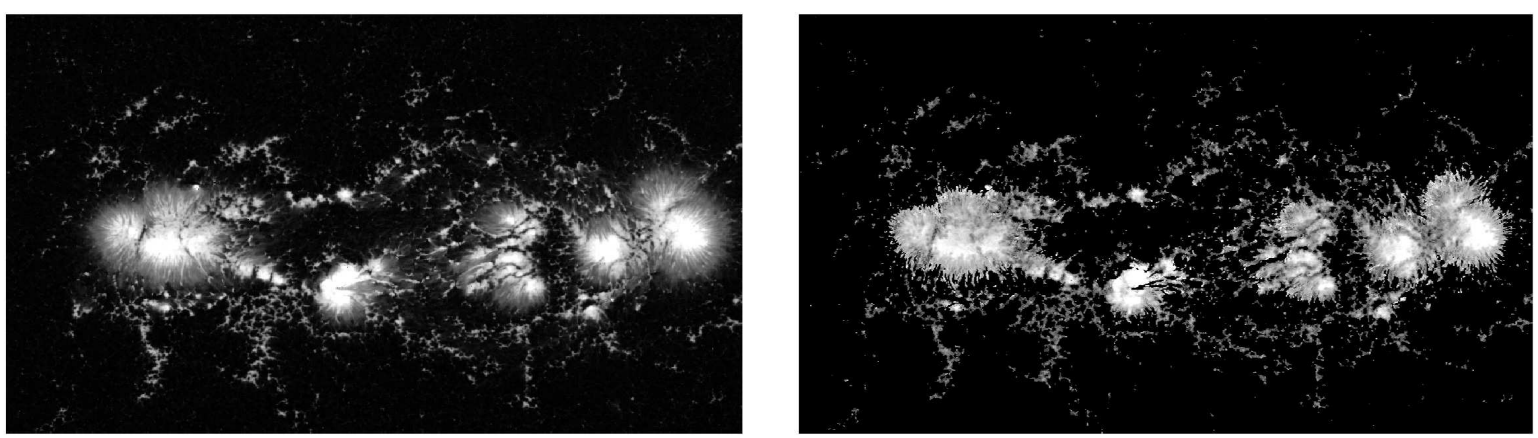

Рис. 6. Магнитограммы модуля вектора магнитного поля АO NOAA 12645, полученные группой обсерватории НАО (слева) и в данной работе (справа). Наблюдения проводились 1 апреля 2017 года в 11:00 UT. Поле зрения магнитограмм $250^{\prime \prime} \times 150^{\prime \prime}$. Черный цвет соответствует величине поля 0 Гс, белый цвет -2000 Гс
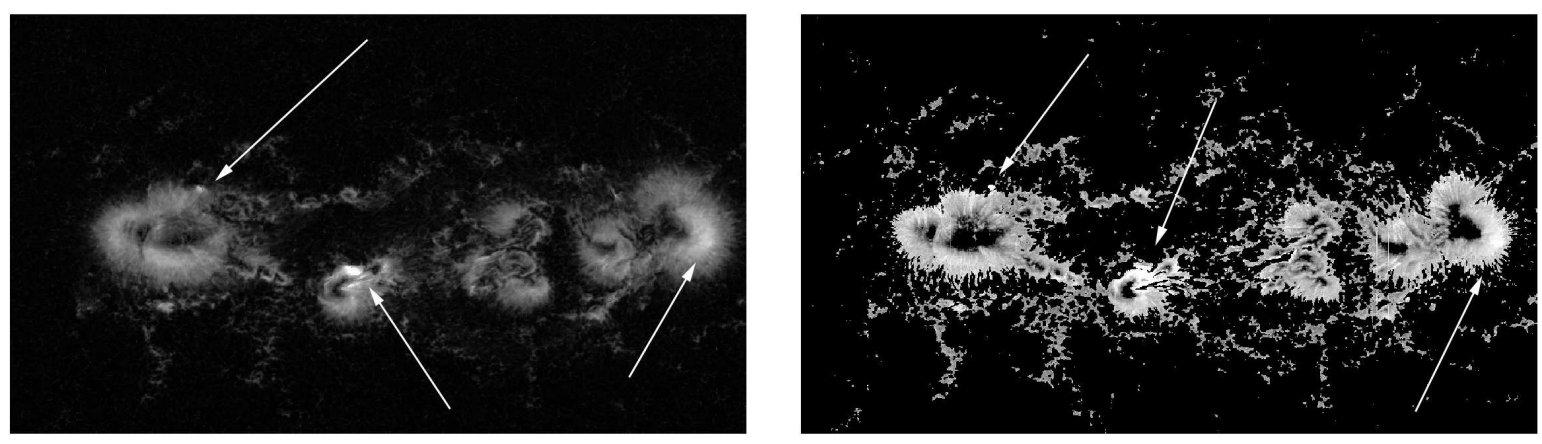

Рис. 7. То же, что на рис. 6, только для модуля поперечного поля. Стрелками показаны локальные усиления поперечного магнитного поля, которые можно выделить на обеих магнитограммах

\section{4 Выводы}

В данной работе мы предложили простой метод для оценки абсолютной величины вектора магнитного поля только из наблюдаемых $I$ и $V$ параметров Стокса. Метод основан на измерении расщепления спектральных компонент в зеемановском мультиплете, который может быть оценен как разность между длинами волн экстремумов профиля $V$ параметра Стокса.

В работе использовались наблюдения внеатмосферного инструмента Hinode/SOT-SP. Для тестирования метода мы воспользовались результатами наблюдений двух АО - NOAA 12172 и 12645. Магнитограммы первой из двух АО позволили нам определить значения калибровочных констант, которые затем применялись для восстановления магнитных полей второй тестовой АО.

Результаты наших расчетов показывают хорошее согласие с измерениями магнитных полей, выполняемых командой обсерватории НАО. Описанный нами метод позволяет также построить приближенные карты модуля полного вектора и модуля поперечного магнитного поля.

Основным недостатком метода, по-нашему мнению, является слишком грубое определение величины расщепления спектральных компонент. Мы полагаем, что использование наблюдаемых профилей $I+V$ и $I-V$ вместо профиля параметра $V$ может дать более точную оценку этой величины, и, соответственно, увеличить точность определения $|\mathbf{B}|$, что будет предметом нашей следующей работы.

Мы надеемся, что после должного усовершенствования метод может найти применение также в области измерения магнитных полей звезд. Он может применяться как дополнительный метод для оценки абсолютной величины магнитного поля звезды как поля магнитного диполя (модуль 
вектора), а также для определения угла $\theta$ между осью магнитного диполя звезды и направлением на наблюдателя.

Благодарности. Авторы благодарны анонимному рецензенту, чьи вопросы и замечания помогли существенно улучшить статью. Работа выполнена при поддержке гранта РНФ (Проект 18-12-00131).

\section{Литература}

Бекерс (Beckers J.M.) // Solar Phys. 1969. V. 9. P. 372.

Брантс (Brants J.J.) // Solar Phys. 1985. V. 95. P. 15.

Бэбкок (Babcock H.W.) // Astrophys. J. 1961. V. 133. P. 572.

Дегл’Инносенти (Landi Degl'Innocenti E.) // Solar Phys. 1982. V. 77. P. 285.

Дегл'Инносенти и Ландолфи (Degl'Innocenti E.L., Landolfi M.) // Kluwer Acad. Publ. 2004. P. 397.

Йео и др. (Yeo K.L. et al.) // Phys. Rev. Lett. 2017. V. 119. 091102.

Лайтс и др. (Lites B. et al. ) // Memorie della Societa Astronomica Italiana. 2007. V. 78. P. 148.

Лайтс и др. (Lites B.W. et al.) // Solar Phys. 2013. V. 283. P. 579.

Лейтон (Leighton R.B.) // Astrophys. J. 1969. V. 156. P. 1.

Нельсон и др. (Nelson N.J. et al.) // Astrophys. J. 2013. V. 762. P. 20.

Паркер (Parker E.N.) // Astrophys. J. 1955. V. 121. P. 491.

Рэйроль (Rayrole J.) // Annales d'Astrophysique. 1967. V. 30. P. 257.

Соланки и Стэнфло (Solanki S.K., Stenflo J.O.) // Astron. Astrophys. 1985. V. 148. P. 123.

Ульрих и др. (Ulrich R.K. et al.) // Astrophys. J. Suppl. Ser. 2002. V. 139. P. 259.

Фан, Фань (Fan Y., Fang F.) // Astrophys. J. 2014. V. 789. P. 13.

Хейл (Hale G.E.) // Astrophys. J. 1908. V. 28. P. 315. 